\title{
Humidity-Modulated Core-Shell Nanopillars for Enhancement of Gecko-Inspired Adhesion
}

Di Tan ${ }^{\dagger \S}$, Aoyi Luo\&§, Xin Wang ${ }^{\dagger}$, Zhekun Shi ${ }^{\dagger}$, Yifeng Lei ${ }^{\dagger}$, Martin Steinhart ${ }^{\#}$, Alexander Kovalev ${ }^{\ddagger}$, Stanislav N. Gorb ${ }^{\ddagger}$ Kevin T. Turner ${ }^{\&}$, Longjian Xue ${ }^{\dagger *}$

${ }^{\dagger}$ School of Power and Mechanical Engineering, The Institute of Technological Science, Wuhan University, South Donghu Road 8, 430072 Wuhan, China

\& Department of Mechanical Engineering and Applied Mechanics, University of Pennsylvania, 220 S. 33rd Street, 19104-6315 Philadelphia, United States

\# Institut für Chemie neuer Materialien, Universität Osnabrück, Barbarastr. 7, 49069 Osnabrück, Germany

* Functional Morphology and Biomechanics, Zoological Institute, Kiel University, Am Botanischen Garten 9, 24098 Kiel, Germany

*E-mail for L.X.: xuelongjian@whu.edu.cn. 


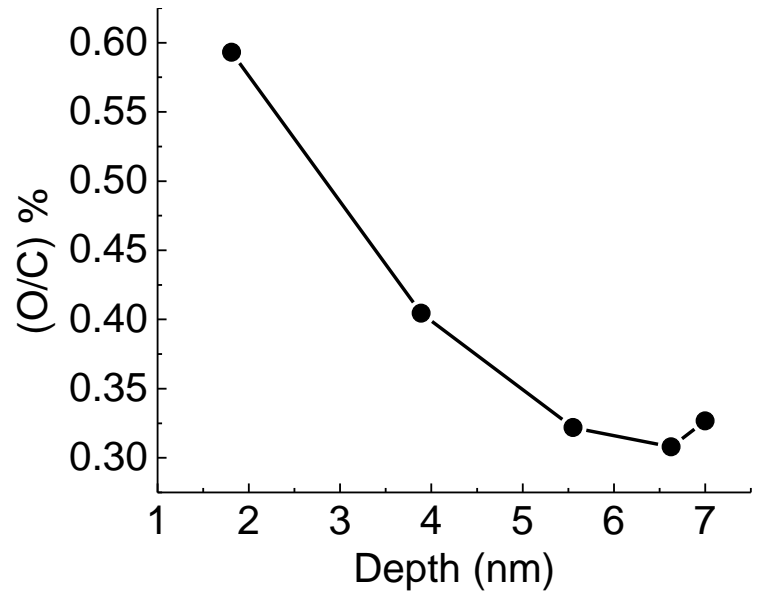

Figure S1. The ratio between oxygen and carbon atoms on the oxygen plasma-treated PS surface in different depths from the very top surface, measured by XPS. 


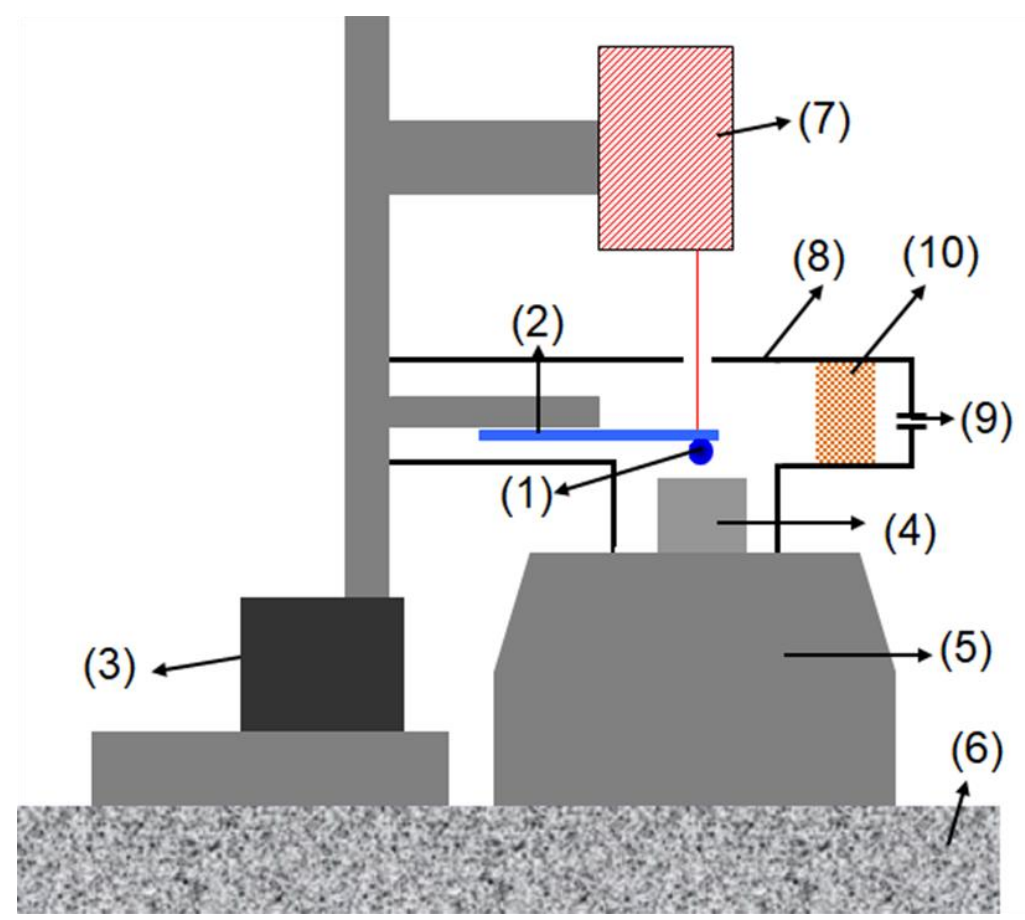

Figure S2. Custom adhesion test system: A sapphire sphere (1) with diameter of $3 \mathrm{~mm}$ glued on the free end of a double-leaf metal spring (2) was used as the probe. The sapphire probe can be moved up and down by a piezoactuator (3). The samples (4) were located on a hexapod nanopositioning stage (5) which is mounted on an anti-vibration table (6). Laser interferometer (7) monitors the deflection of metal spring during the loading and unloading, which could be converted into detecting forces. Measurements were carried out inside a humidity chamber (8) at room temperature under controlled humidity conditions. The humidity was controlled by piping gas flow with different RH into the chamber via gas inlet valve (9). Sponge (10) was used to homogenize the gas flow in the humidity chamber. 

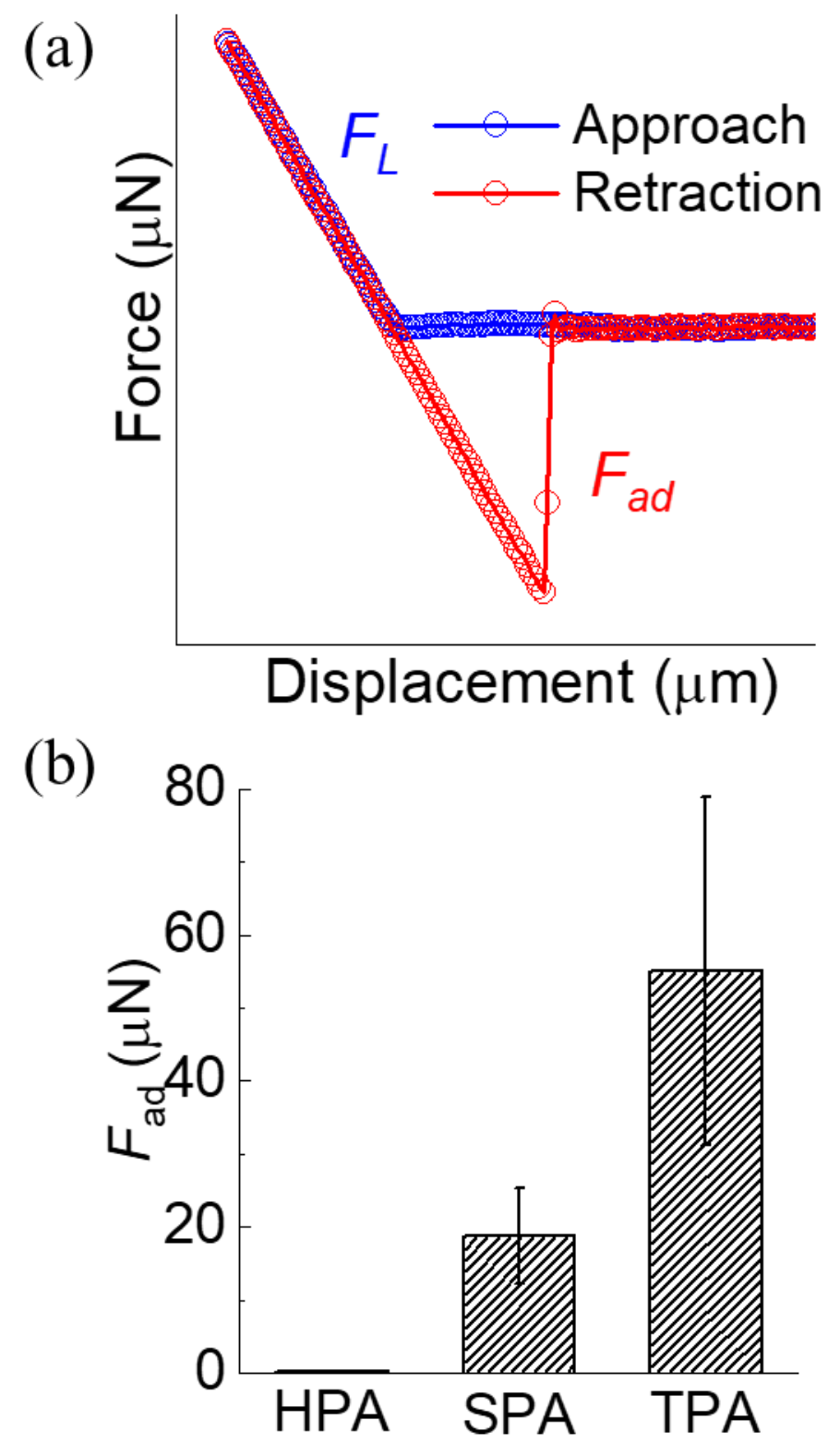

Figure S3. (a) Typical adhesion measurement curve; (b) $F_{a d}$ of HPA, SPA and TPA at RH of $35 \%$. 
(a)

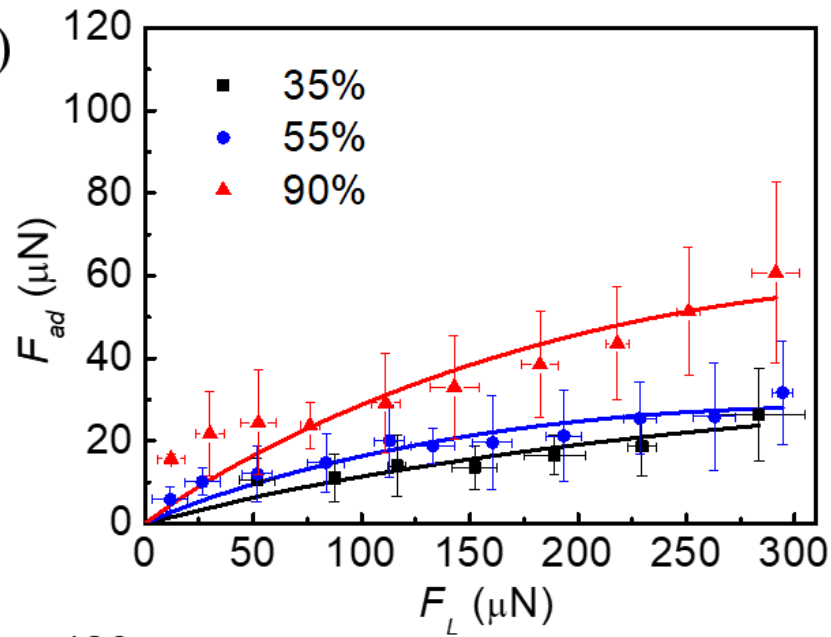

(b)

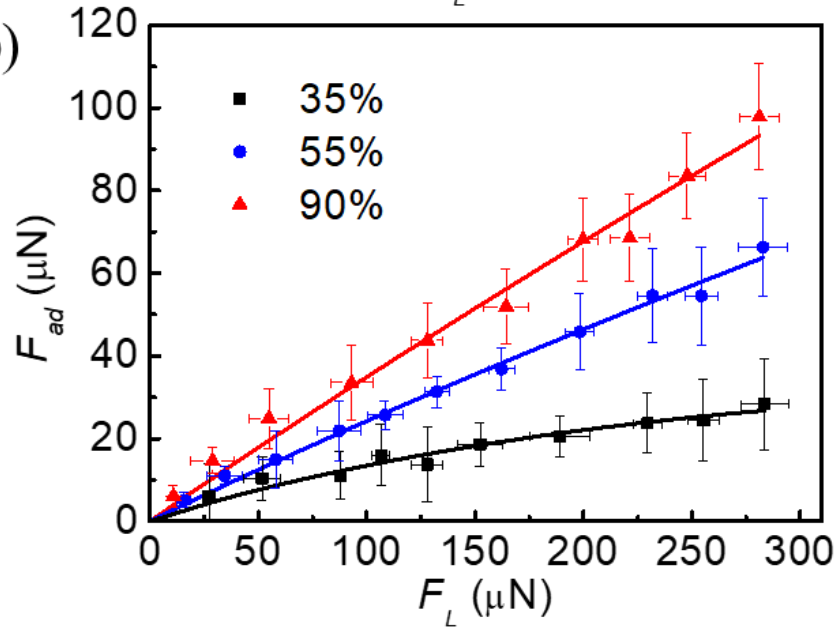

Figure S4. Loading dependence of adhesion $\left(F_{\mathrm{ad}}\right)$ of (a) hydrophobic and (b) hydrophilic SPA at different humidity. 


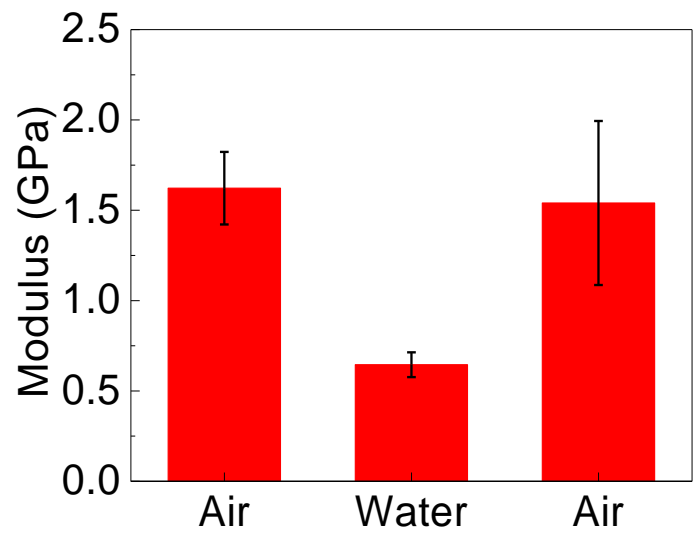

Figure S5. The superficial modulus of PS films in air, under water and after drying for $12 \mathrm{~h}$. 


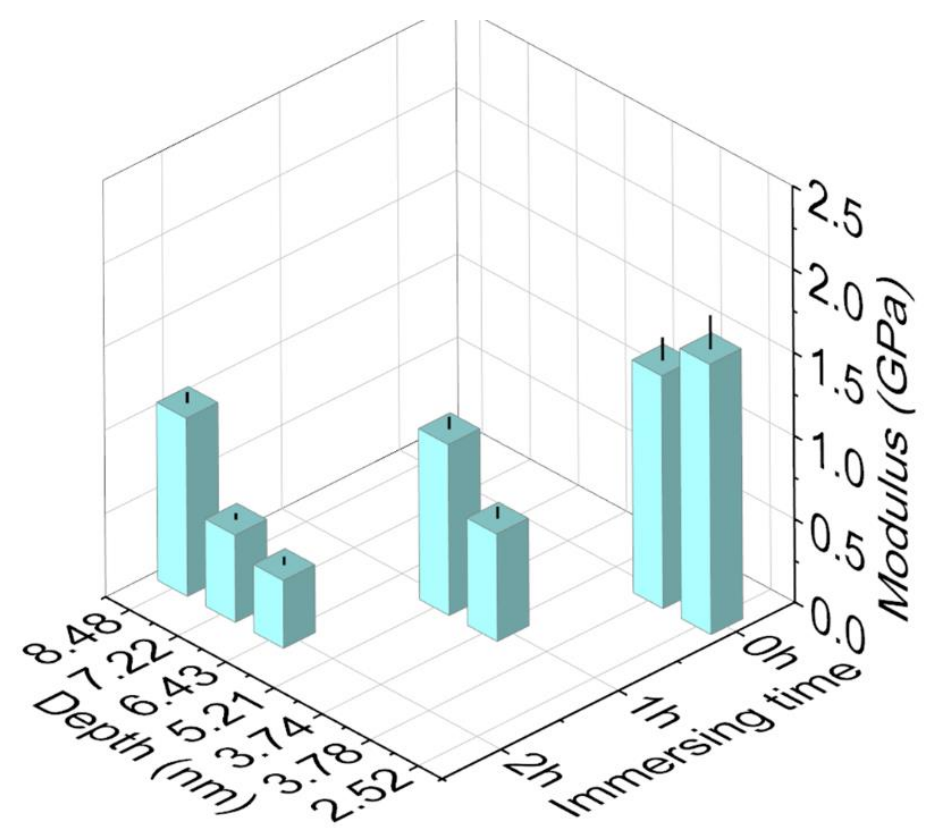

Figure S6. The superficial modulus of PS films under water with different immersing time detected with different loading forces 


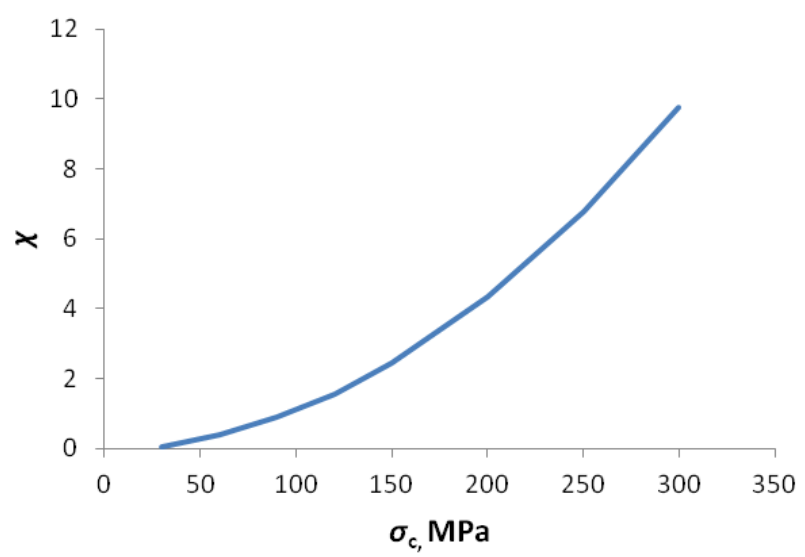

Figure S7. $\chi$ as a function of $\sigma_{\mathrm{c}}$

The selections of those parameters are based on the considerations as follows: Work of adhesion, $\gamma$, of polystyrene (PS) is assumed to be around $30 \mathrm{~mJ} / \mathrm{m}^{2}{ }^{1}$ Typically, adhesion strength $\sigma_{c}$ of a material is estimated to be $1 \%$ to $10 \%$ of its modulus, ${ }^{2,3}$ so $\sigma_{c}$ could range from $30 \mathrm{MPa}$ to $300 \mathrm{MPa}$. Then the critical separation $\delta_{c}=2 \gamma / \sigma_{c}$ with linear traction-separation law ranges from $2 \mathrm{~nm}$ to $0.2 \mathrm{~nm}$. According to Tian et al., ${ }^{2}$ the pulloff stress of fibrillar adhesives depends on a dimensionless parameter $\chi=\left(\sigma_{\mathrm{c}} R\right) /\left(2 \pi E^{*} \delta_{c}\right)$. When $\chi$ is smaller than 1, the pull-off stress is independent of the fiber geometry and the interfacial stress distribution, and it reaches its theoretical maximum $\sigma_{\mathrm{c}}$. When $\chi$ is larger than 1, the pull-off stress is affected by the fiber geometry and the interfacial stress distribution, and it decreases relative to $\sigma_{\mathrm{c}}$ as $\chi$ increases. From the experiments results shown in Fig. 2c, the measured pull-off force of TPA is more than 2 times larger than SPA, which means the pull-off stress is very sensitive to fiber tip geometry. The significant difference in measured adhesion between TPA and SPA indicates that $\chi$ is significantly larger than 1 . Figure $\mathrm{S} 7$ shows $\chi$ as a function of $\sigma_{\mathrm{c}}, \chi=1$ when $\sigma_{\mathrm{c}}=100 \mathrm{MPa}$, so $\sigma_{\mathrm{c}}$ should at least on $100 \mathrm{MPa}$ scale. $\sigma_{\mathrm{c}}$ is chosen to be $300 \mathrm{MPa}$ and $\delta_{c}=0.2 \mathrm{~nm}$ accordingly in the simulation which gives $\chi=9.8$ to theoretically allow significant adhesion difference between TPA and SPA.

\section{References:}

(1) Brandrup, J.; Immergut, E.; Grulke, E. Polymer Handbook, 4th ed.; Wiley: New York, 1999.

(2) Tang, T.; Hui, C.-Y.; Glassmaker, N. J. Can a fibrillar interface be stronger and tougher than a non-fibrillar one? J. R. Soc. Interface 2005, 2, 505-516.

(3) Gao, H.; Chen, S. Flaw tolerance in a thin strip under tension. J. Appl. Mech. 2005, 732-737. 


\section{Calibration of AFM probes:}

Before the force measurements, the probe was calibrated by doing indentation on a quartz glass slide. As quartz has a large modulus of $\sim 72 \mathrm{GPa}$, the detected deformation solely comes the deformation of the probe. The spring constant of the probe was then calibrated automatically by the AFM software from the indentation curve. The calibrated spring constant is $0.229 \mathrm{~N} / \mathrm{m}$. 